\title{
Complete and variant forms of the 'gonococcal genetic island' in Neisseria meningitidis
}

Correspondence
Nigel J. Saunders
Nigel.Saunders@path.ox.ac.uk

Received 27 January 2005

Revised 24 August 2005

Accepted 25 August 2005

\author{
Lori A. S. Snyder, ${ }^{1}$ Stephen A. Jarvis ${ }^{2}$ and Nigel J. Saunders ${ }^{1}$ \\ 'Bacterial Pathogenesis and Functional Genomics Group, Sir William Dunn School of \\ Pathology, University of Oxford, Oxford OX1 3RE, UK \\ ${ }^{2}$ Department of Computer Science, University of Warwick, Coventry CV4 7AL, UK
}

\begin{abstract}
Comparative genome hybridization using the pan-Neisseria microarray identified genes from the gonococcal genetic island (GGI) within Neisseria meningitidis strains of serogroups W-135, $\mathrm{H}$, and $\mathrm{Z}$. While some of these strains contain nearly all of the genes of the GGI, there are differences in the presence of some of these genes between the strains, including between those of the same serogroup. Attempts were then made to determine the location of the GGI in these meningococci. Sequencing of Neisseria gonorrhoeae strain MS11 revealed that the GGI is a conjugative plasmid that can be chromosomally integrated at the dif sites near ung and can also be present in its circularized form. In N. meningitidis, a dif site is present in this location and also serves as the point of chromosomal integration of the GGI in this species.
\end{abstract}

\section{INTRODUCTION}

In Neisseria gonorrhoeae strain MS11, a large section of sequence has been identified that is not present in the $N$. gonorrhoeae genome sequence of strain FA1090 (Dillard \& Seifert, 2001; Hamilton et al., 2005). This was identified following the investigation of a strain-specific serum resistance gene (McShan et al., 1987), an associated peptidoglycan hydrolase (Dillard \& Seifert, 1997), and sequencing of the associated $57 \mathrm{~kb}$ flanking sequences, which are present in most, but not all, gonococcal strains (Dillard \& Hamilton, 2002; Hamilton et al., 2005). Due to this element's previously reported absence from Neisseria meningitidis, it has been called the 'gonococcal genetic island' (GGI) (Dillard \& Seifert, 2001; Dillard \& Hamilton, 2002). Our annotation of this sequence, provided by Joe Dillard for use in the construction of the pan-Neisseria microarray (Snyder et al., 2004), includes 57 potential coding sequences. The sequence has many features that indicate horizontal acquisition of the region, including atypical $\mathrm{G}+\mathrm{C}$ content, and flanking sequences similar to the dif sites recognized by XerCD recombinases in Escherichia coli and Haemophilus influenzae (Dillard \& Hamilton, 2002).

The original pan-Neisseria microarray (Snyder et al., 2004) was designed, constructed, and tested by an international consortium and contains probes representing all coding

\footnotetext{
Abbreviations: CDS, coding sequence; $\mathrm{CGH}$, comparative genome hybridization; GGI, gonococcal genetic island; MLST, multilocus sequence typing; MME, minimal mobile element.

The GenBank/EMBL/DDBJ accession numbers for the sequences reported in this paper are DQ171942, DQ171943, DQ171944, and DQ171945.
}

regions from three neisserial genomes (N. gonorrhoeae strain FA1090, and N. meningitidis strains MC58 and Z2491) and our predicted gene sequences from the GGI of N. gonorrhoeae strain MS11. Probes were designed based upon a comparative analysis of the genomes, so that common probes could be used to assess orthologous genes between the neisserial strains and species. The use of the panNeisseria microarray to assess gene complements in strains other than those used for its design has been published previously for N. gonorrhoeae (Snyder et al., 2004). An extended version of the pan-Neisseria microarray (-v2) has been constructed, which includes additional gene probes based on the $N$. meningitidis strain FAM18 genome sequence, neisserial genes from GenBank/EMBL that are not in any of the genome sequences currently available, and genes identified through our ongoing investigations of minimal mobile elements (MMEs) (Saunders \& Snyder, 2002; Snyder et al., 2004).

We previously reported the presence of the GGI in $N$. gonorrhoeae strain FA19, identified by comparative genome hybridization $(\mathrm{CGH})$ using the pan-Neisseria microarray (Snyder et al., 2004). During a test of the ability of the pan-Neisseria microarray-v2 to generate CGH data from diverse strains of $N$. meningitidis, hybridization to the GGIassociated probes was identified in serogroup $\mathrm{H}$ strain 98/ 250521. Due to previous reports suggesting that GGI genes were restricted to N. gonorrhoeae (Dillard \& Seifert, 2001; Dillard \& Hamilton, 2002), these results were repeated and expanded to include other serogroup $\mathrm{H}$ strains, as well as strains from other serogroups. The microarray hybridization data suggest that these different $N$. meningitidis capsular serogroup strains contain both complete and partial versions of the GGI. 


\section{METHODS}

The pan-Neisseria microarray-v2. The revised and expanded pan-Neisseria microarray (-v2) was designed and generated by using the same strategy as and similar protocols to those described previously for the original pan-Neisseria microarray (Snyder et al., 2004). The pan-Neisseria microarray-v2 contains probes directed to the most conserved regions of the annotated potential coding sequences of $N$. gonorrhoeae strain FA1090 (GenBank accession no. AE004969), N. meningitidis strain MC58 (Tettelin et al., 2000), N. meningitidis strain Z2491 (Parkhill et al., 2000), and N. meningitidis strain FAM18 (ftp://ftp.sanger.ac.uk/pub/pathogens/nm). Probes were also included for additional sequences from the $N$. gonorrhoeae strain MS11 GGI (Dillard \& Seifert, 2001; Dillard \& Hamilton, 2002; Hamilton et al., 2005), neisserial genes from GenBank/EMBL that are not in the genome sequences currently available, genes identified through our ongoing investigations of MMEs (Saunders \& Snyder, 2002; Snyder et al., 2004), and antibiotic resistance cassette markers used in mutagenesis. Each probe of $150-450 \mathrm{bp}$ targets the most conserved ( $>90 \%$ similar) non-repetitive region within each coding sequence, achieving maximal hybridization to orthologous genes in different strains and species. Where possible, significant crosshybridization was eliminated, or noted, to minimize the potential for cross-hybridization with other genes, which would generate false positive results. Because only one sequence of a GGI is currently available, it was not possible to specifically select probes from within the most conserved regions of these coding sequences; therefore, a greater number of weak or false negative results may occur if these genes are divergent. The PCR product probes generated were suspended in Genetix Spotting Solution for Amine slides. The microarray slides were printed with a Genetix QArray Mini microarray printer using $150 \mu \mathrm{m}$ solid aQu pins onto Genetix Amine slides.

Bacterial strains used. Representative strains from serogroups $\mathrm{C}$ (strains SB25 and 2120), W-135 (strain A22), X (strain 860060), Y (strain 860800), and Z (strain E32) were obtained from the MLST (multilocus sequence typing) meningococcal strain collection (http://pubmlst.org/neisseria/). To supplement these, additional strains of atypical serogroups were obtained from the Meningococcal Reference Laboratory [Manchester Public Health Laboratory Service (PHLS), UK]. In all, the CGH data analysed were from N. meningitidis strains MC58 (serogroup B, sequence strain), Z2491 (serogroup A, sequence strain), FAM18 (serogroup C, sequence strain), SB25 (serogroup C, MLST strain), 2120 (serogroup C, MLST strain), A22 (serogroup W-135, MLST strain), 860060 (serogroup X, MLST strain), 860800 (serogroup Y, MLST strain), E32 (serogroup Z, MLST strain), 00/240794 (serogroup D, reference strain NCTC 009714), 01/241825 (serogroup 29E, PHLS strain), 98/250521 (serogroup H, PHLS strain), 97/252572 (serogroup H, PHLS strain), 97/252675 (serogroup H, PHLS strain), 01/241809 (serogroup X, PHLS strain), 01/241693 (serogroup X, PHLS strain), 00/240868 (serogroup Z, PHLS strain), 01/241422 (serogroup Z, PHLS strain), and 01/241471 (serogroup Z, PHLS strain). N. gonorrhoeae strains MS11 and FA19, as strains known to contain the GGI (Snyder et al., 2004), were hybridized to the pan-Neisseria microarray-v2 as controls.

Comparative genome microarray hybridization. Chromosomal DNA was extracted (McAllister \& Stephens, 1993) from the neisserial strains following growth on GC medium (BD) with Kellogg and ferric nitrate supplements (Kellogg et al., 1963) at $37^{\circ} \mathrm{C}$, in $5 \%(\mathrm{v} / \mathrm{v}) \mathrm{CO}_{2}$ overnight. DNA $(20 \mu \mathrm{g})$ was fluorescently labelled through direct incorporation of FluoroLink Cy3-dCTP or FluoroLink Cy5-dCTP (Amersham Biosciences) using 5 units DNA polymerase I Klenow fragment (Bioline) and $3 \mu \mathrm{g}$ random hexamer primers (Invitrogen). Unincorporated nucleotides and random primers were removed using QIAquick Nucleotide Removal columns
(Qiagen) according to the manufacturer's instructions. Labelled DNA-DNA probe microarray hybridizations were conducted in $4 \times$ SSC, $0 \cdot 29 \%$ SDS under LifterSlips (Erie Scientific) at $65^{\circ} \mathrm{C}$ overnight. The slides were washed at $65^{\circ} \mathrm{C}$ in $1 \times$ SSC, $0.05 \%$ SDS for $2 \mathrm{~min}$, at room temperature in $0.06 \times \mathrm{SSC}$ for $2 \mathrm{~min}$, and in fresh $0.06 \times$ SSC for 2 min before drying by airbrush (Paasche, with SimAir compressor). Microarray slides were scanned using a ScanArray Express HT Microarray Scanner (Perkin Elmer) and analysed using ScanArray Express software v2.1 (Perkin Elmer).

Each strain was compared against two other strains in a three-way experimental design, such that strains $\mathrm{A}, \mathrm{B}$, and $\mathrm{C}$ were compared as $A$ vs $B, B$ vs $C$, and $C$ vs $A$. The presence or absence of any gene in one pairwise analysis was validated by comparison to the results of the unrelated comparative hybridization. If necessary, hybridizations were repeated to resolve ambiguities.

Dinucleotide signature analysis and $\mathbf{G}+\mathbf{C}$ content determination. Gene-by-gene dinucleotide signature analysis has previously been applied to the complete genome sequence of $N$. meningitidis strain MC58 (Tettelin et al., 2000) and to the genes of the neisserial $d c w$ cluster in N. gonorrhoeae (Snyder et al., 2001). A revised version of this methodology was used for this study, which compensates for the sampling effects of addressing variable gene lengths that tend to artefactually increase the divergence of shorter sequences (Saunders et al., 2005). The variation in the signature of each individual coding region, in comparison to the signature of the genome as a whole, can indicate genes that have been acquired from other species with different compositional biases. Dinucleotide signatures were determined for the complete genome of $N$. gonorrhoeae strain FA1090 for reference, for each of the coding regions of this genome, and for the N. gonorrhoeae strain MS11 GGI (GenBank accession no. AY803022). The deviation from the mean genome signature of each coding sequence was determined and compared against the mean divergence of all annotated coding sequences in the genome.

The percentage of $\mathrm{G}$ and $\mathrm{C}$ bases within each gene was determined and compared with the $\mathrm{G}+\mathrm{C}$ content of the $N$. gonorrhoeae strain FA1090 genome sequence.

GGI location determination using microarrays. The determination of the location of the GGI was attempted using a modification of the CGH protocol described above. Specific primers that pointed out from the GGI (Table 1) were used instead of random primers. Primers at the GGI01 end of the GGI were used with FluoroLink Cy3-dCTP, and primers at the GGI56/GGI57 end of the GGI were used with FluoroLink Cy5-dCTP (Amersham Biosciences). All other conditions and methods were identical to those used for CGH.

Table 1. Oligonucleotide primers used in this study

\begin{tabular}{|c|c|}
\hline Primer & Sequence \\
\hline GGI01out1 & 5'-CTACAGCAATTCGATACTACG-3' \\
\hline GGI01out2 & 5'-GAAGTTTACAGACTAAGCCGG-3' \\
\hline GGI56out1 & 5'-GTGCAGGCTCAAGGTTGC-3' \\
\hline GGI56out2 & $5^{\prime}$-ATTGGCAACGACATTAACATGC-3' \\
\hline GGI57out3 & 5'-AACTGCACCCTGAGTATCG-3' \\
\hline GGIdelF & 5'-ATGCGGCCAAAATCTATACC-3' \\
\hline GGIdelR & 5'-ACAGCAGCCGTATTTTCAGG-3' \\
\hline GGIRjoinFA & 5'-GCGTGTCAGGTTCATTTGGC-3' \\
\hline GGIRjoinMS & 5'-AACATCCGCAATCGAACAGG-3' \\
\hline WGGIdifR & 5'-ATGCCCACCTTGAAAATATGG-3' \\
\hline
\end{tabular}


GGI mutant construction. To attempt to construct whole island knock-out mutants in the GGI-containing $N$. meningitidis strains, the N. gonorrhoeae strain FA1090 region was PCR amplified using $P f u$ Turbo polymerase (Stratagene) with primers GGIdelF and GGIdelR (Table 1) and cloned into pCR-Script-Cam (Stratagene). This region of $2003 \mathrm{bp}$ contains a portion of the genes that flank the GGI in gonococcal strain MS11 (ung and XNG0740, see Fig. 1). Through blunt-end digestion of the pCR-Script-Cam vector, with EcoRV and SmaI, and religation, the EcoRI site in the multiple cloning site of the vector was removed. The cloned product contains a hypothetical gene (XNG0741) with naturally occurring EcoRI sites just inside the coding region at each end, which were used to insert one of two antibiotic resistance cassettes. A construct was generated containing a kanamycin resistance cassette from pCR2.1 (Invitrogen) fused to the neisserial $r e c A$ promoter and a second construct was made using the kanamycin resistance determinant aphA-3, which lacks a promoter and reduces the likelihood of polar effects (Ménard et al., 1994). These plasmids were named pGGIdelP recakan and pGGIdelaphA-3. PCR amplicons generated using a 1:1000 dilution of the plasmids were used to transform N. meningitidis strain 98/250521, as well as N. gonorrhoeae strains FA1090 and FA19 as controls. Transformants were selected on GC agar containing 50 mg kanamycin $1^{-1}$.
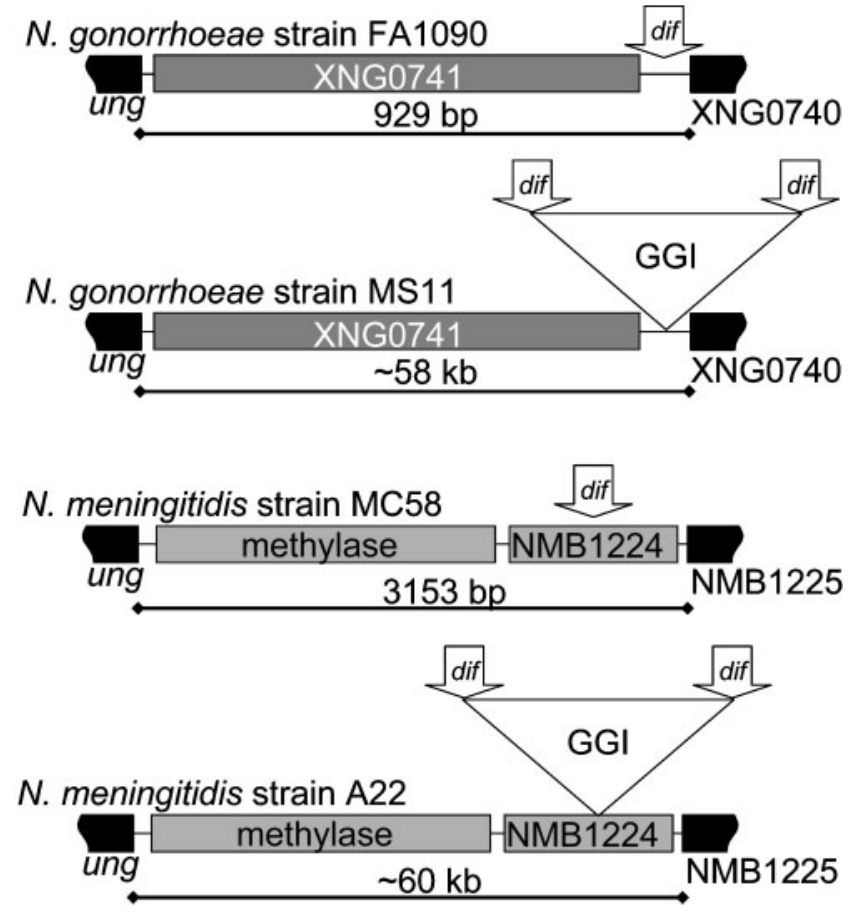

Fig. 1. The MMEung and the integration of the GGl at the dif site. The MME region adjacent to ung has been described previously (Snyder et al., 2004). It is now apparent, however, from $\mathrm{CGH}$ and sequencing data, that the GGl present in N. gonorrhoeae strain MS11 has inserted at the dif site between two hypothetical genes. This event is not in accordance with the MME model; it is rather an insertion into an existing MME region. In $N$. meningitidis strain MC58, the MMEung contains a methylase and NMB1224, rather than the hypothetical gene XNG0741 present in gonococcal strains FA1090 and MS11. The annotated coding region NMB1224 contains a dif site, which is the point of integration of the GGI in N. meningitidis strain A22 (GenBank accession no. DQ171942).
Sequencing of GGI ends. PCR amplifications from strain MS11 used HotStarTaq polymerase (Qiagen) and primer pairs GGIout1 with GGIdelF, GGIout1 with GGI57out3, and GGI57out3 with GGIdelR (Table 1). The product from N. gonorrhoeae strain MS11 generated using primer pair GGI01outl with GGIdelF was sequenced directly, whereas the other two strain MS11 products were TOPO TA cloned (Invitrogen) for subsequent sequencing using primers M13 Forward and M13 Reverse (Invitrogen). Primer pair GGIRjoinMS with GGIjoinFA was used to generate a new PCR amplicon that was internal to the strain MS11 GGI57out3/GGIdelR product, which was then sequenced using these primers. A PCR product spanning the meningococcal strain A22 dif site was generated using GGI56outl and WGGIdifR, which was designed using the NMB1224 sequence from N. meningitidis strain MC58. This spanned the junction between the chromosomal sequence and the GGI sequence. The product was TOPO TA cloned (Invitrogen) and sequenced using M13 Forward and M13 Reverse (Invitrogen). Sequencing used ABI Prism BigDye Terminator Cycle Sequencing version 3.0 (Applied Biosystems), and was resolved on an ABI Prism 3100 DNA Sequencer (Applied Biosystems). Sequences were analysed using Trev from the Staden package (Bonfield et al., 2002) and the Wisconsin Package from GCG (Accelrys). Sequence data were deposited into GenBank under accession numbers DQ171942, DQ171943, DQ171944, and DQ171945.

\section{RESULTS}

\section{Comparative genome microarray hybridizations}

Controls. Each microarray was assessed for the presence of the genes of the GGI, as determined by hybridization to the printed probes. Hybridizations with the sequenced meningococcal strains (MC58, Z2491, and FAM18) were conducted as negative controls; these did not indicate the presence of the GGI-associated genes. Positive control hybridizations were performed using $N$. gonorrhoeae strains MS11 and FA19, which are known to contain the GGI (Dillard \& Seifert, 2001; Snyder et al., 2004). Four of the GGI probes do not consistently generate a signal, although the cause for this is unclear. These probes (for GGI09, GGI43, GGI45, and GGI48) generated clear and bright PCR product bands during microarray production. Additionally, our recent reannotation of the GGI includes a coding sequence (CDS) that was previously unannotated for which no probe has been designed (GGI46). Probes were not made to the repeated sequences flanking GGI26 (sequences annotated as GGI25 and GGI27).

To verify that each of the tested strains was correctly identified as N. meningitidis, the microarray results were assessed for the presence of genes such as the ctr (NMB0071, NMB0072, NMB0073, and NMB0074) and lip (NMB0082 and NMB0083) capsule biosynthesis genes and $o p c$ (NMB1053). In all cases, these characteristic meningococcal genes were present in the tested meningococcal strains.

Serogroup H. A near complete version of the GGI, with 47 of the 50 probed GGI annotated coding regions, is present in N. meningitidis strain 98/250521 (Table 2). Two other serogroup $\mathrm{H}$ strains were assessed: $N$. meningitidis strains 97/252572 and 97/252675 (Table 2). Strain 
Table 2. Comparative genome hybridization to GGI probes

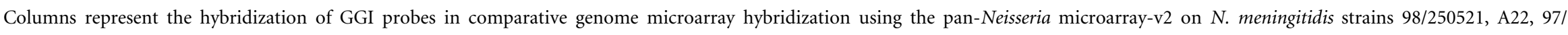

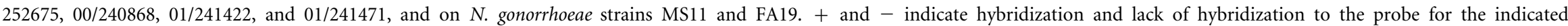
GGI sequence. Gene numbers are from the annotation of GenBank accession no. AY803022 (GGI gene) and from our own annotation of this sequence (CDS).

\begin{tabular}{|c|c|c|c|c|c|c|c|c|c|}
\hline $\begin{array}{l}\text { GGI } \\
\text { gene }\end{array}$ & CDS & Annotation & $\begin{array}{c}98 / 250521 \\
\text { serogroup } \\
\text { H }\end{array}$ & $\begin{array}{c}\text { A22 } \\
\text { serogroup } \\
\text { W135 }\end{array}$ & $\begin{array}{c}97 / 252675 \\
\text { serogroup } \\
\text { H }\end{array}$ & $\begin{array}{c}00 / 240868 \\
\text { serogroup } \\
Z\end{array}$ & $\begin{array}{c}01 / 241422 \\
\text { serogroup } \\
Z\end{array}$ & $\begin{array}{c}01 / 241471 \\
\text { serogroup } \\
Z\end{array}$ & $\begin{array}{l}\text { MS11 } \\
\& \\
\text { FA19 }\end{array}$ \\
\hline NG5001 & GGI01 & traD, channelling/conjugative DNA transfer protein & + & + & + & + & + & + & + \\
\hline NG5002 & GGI02 & traI, helicase/nickase/conjugative relaxase & + & + & + & + & + & + & + \\
\hline NG5003 & GGI03 & Hypothetical & + & + & + & + & + & + & + \\
\hline NG5004 & GGI04 & iagB, transglycosylase/similar to coliform pilT & + & + & + & + & + & + & + \\
\hline NG5005 & GGI05 & Hypothetical & + & + & + & + & + & + & + \\
\hline NG5006 & GGI06 & Hypothetical & + & - & + & + & + & + & + \\
\hline NG5007 & GGI07 & traL, pilus assembly protein & + & + & + & + & + & + & + \\
\hline NG5008 & GGI08 & traE, pilus assembly protein & + & + & - & + & + & - & + \\
\hline NG5010 & GGI10 & $\operatorname{tr} a B$, pilus assembly protein & + & + & - & - & - & + & + \\
\hline NG5011 & GGI11 & $d s b C$, thiol-disulphide interchange protein & + & + & - & - & - & + & + \\
\hline NG5012 & GGI12 & $h t d D / t r a V$, pilus assembly protein & + & + & - & - & - & + & + \\
\hline NG5013 & GGI13 & traC, pilus assembly protein & + & + & - & - & - & + & + \\
\hline NG5014 & GGI14 & Hypothetical & + & - & - & - & - & + & + \\
\hline NG5015 & GGI15 & traFl, pilus assembly protein & + & + & - & - & - & + & + \\
\hline NG5016 & GGI16 & traW, pilus assembly protein & + & + & - & - & - & + & + \\
\hline NG5017 & GGI17 & $\operatorname{tra} U$, pilus assembly protein & + & + & - & - & - & + & + \\
\hline NG5018 & GGI18 & trbC, pilus transfer protein & + & + & - & - & - & + & + \\
\hline NG5019 & GGI19 & $\operatorname{traN}$, frameshifted mating stabilization protein & + & + & - & - & - & + & + \\
\hline NG5021 & GGI20 & Hypothetical & + & + & - & - & - & + & + \\
\hline NG5022 & GGI21 & traF2, pilus assembly protein & + & + & - & - & - & + & + \\
\hline NG5023 & GGI22 & traH, pilus assembly protein & + & + & - & - & - & + & + \\
\hline NG5024 & GGI23 & traG, self-annotation from Q93NF1 & + & + & - & - & - & + & + \\
\hline NG5025 & GGI24 & atlA, bacteriophage lysozyme/lysin & - & - & - & - & - & - & + \\
\hline NG5028 & GGI26 & $\operatorname{csp} A$, cold shock protein & + & + & - & - & - & + & + \\
\hline NG5030 & GGI28 & Hypothetical & + & + & - & - & - & + & + \\
\hline NG5031 & GGI29 & Hypothetical & + & + & + & + & + & + & + \\
\hline NG5032 & GGI30 & Conserved hypothetical & + & + & + & + & + & + & + \\
\hline NG5033 & GGI31 & $p r l F, h t r A$ suppressor protein & + & + & + & + & + & + & + \\
\hline NG5034 & GGI32 & Hypothetical & + & + & + & + & + & + & + \\
\hline NG5035 & GGI33 & Frameshifted conserved hypothetical protein & + & + & + & + & + & + & + \\
\hline NG5036 & GGI34 & Hypothetical & + & + & + & + & + & + & + \\
\hline NG5037 & GGI35 & Hypothetical & + & + & + & + & + & + & + \\
\hline NG5038 & GGI36 & Hypothetical & + & + & + & + & + & + & + \\
\hline NG5039/NG5040 & GGI37 & Frameshifted restriction methylase & + & + & + & + & + & + & + \\
\hline
\end{tabular}




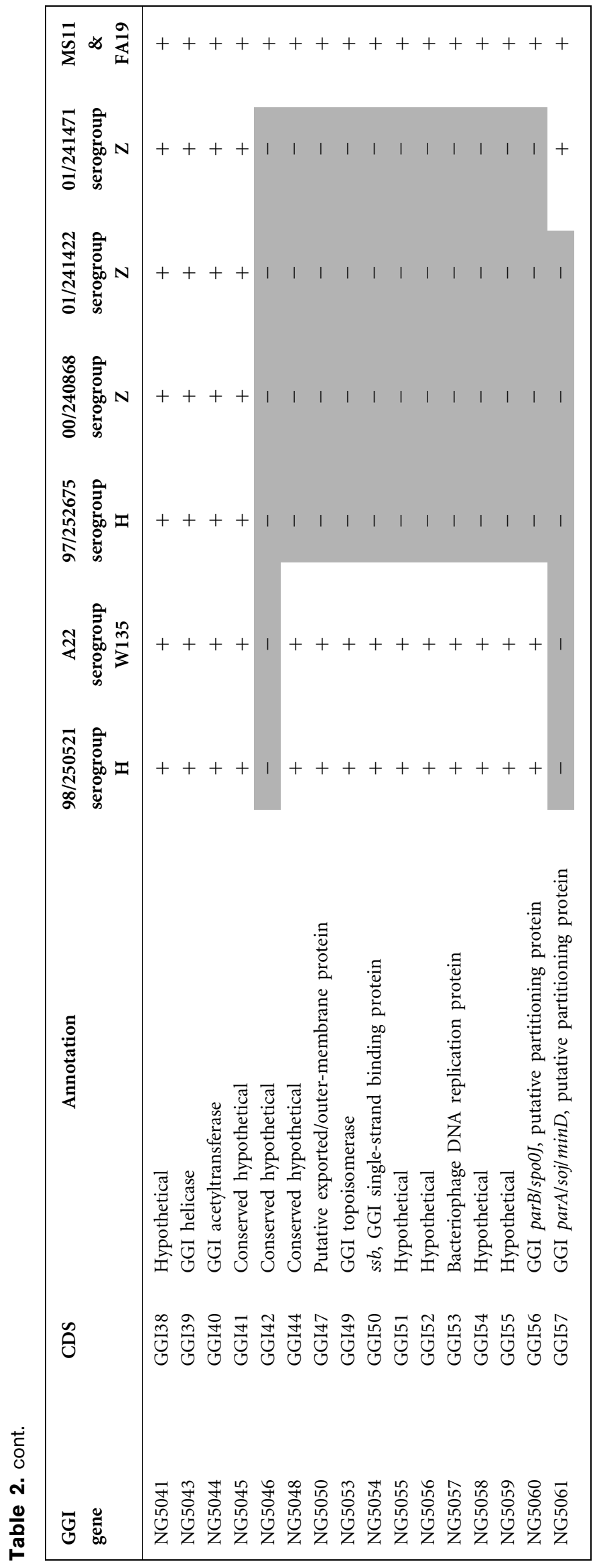

97/252572 did not hybridize to the GGI probes. Strain 97/ 252675 hybridized to some of the probes, indicating that portions of the GGI (GGI01-GGI07 and GGI29-GGI41) are present, but large contiguous sections appear to be absent.

Serogroup W-135. One serogroup W-135 strain was assessed, N. meningitidis strain A22 (Table 2). The GGI probe hybridization pattern was very similar to that of the serogroup H strain 98/250521, which contains a near complete GGI. Forty-five of the 50 GGI genes interrogated were present in strain A22.

Serogroup Z. Four strains of N. meningitidis serogroup Z were assessed: E32, 01/241471, 00/240868, and 01/241422 (Table 2). Strain E32 did not hybridize to the GGI probes. Strains $00 / 240868$ and $01 / 241422$ have identical GGI probe hybridization profiles, which are similar to that of serogroup H strain 97/282675, containing GGI01-GGI08 and GGI29-GGI41. The most complete GGI of those identified in the serogroup Z strains is in strain 01/241471, although GGI42-GGI56 are apparently absent. Other GGI genes are also absent or divergent in this strain, while one of the hybridized probes is not hybridized by any of the other meningococci assessed in this study (GGI57).

Other meningococcal strains. Of the combined MLST/ PHLS strain set, strains of serogroups C (strains SB25 and 2120), D (strain 00/240794), 29E (strain 01/241825), $\mathrm{X}$ (strains 860060, 01/241809, and 01/241693), and Y (strains O929 and 860800) did not hybridize with GGIassociated probes.

\section{Dinucleotide signature analysis and $\mathrm{G}+\mathrm{C}$ content}

The dinucleotide signatures and $\mathrm{G}+\mathrm{C}$ content of the predicted coding regions of the GGI are supportive of the horizontal acquisition origin of some genes and segments of the GGI. The weighted deviation of the dinucleotide signature and the $\mathrm{G}+\mathrm{C}$ content were calculated for each of the 57 coding regions (Fig. 3). The mean divergence of the gonococcal genes in the FA1090 genome is $0.062 \%$, with an SD of 0.021 and $95 \%$ confidence intervals of 0.0009 . Compared with the FA1090 genes, GGI23 (traG) is the second most divergent gene not encoded by a repeat (a different source of signature divergence) assessed. These results show that several components of the GGI have sequence characteristics suggesting that they are not neisserial in origin. However, some regions may not have a foreign source, and/or have been in the neisseriae sufficiently long to have ameliorated to a typical neisserial composition.

\section{Determination of GGI chromosomal location by microarray hybridization}

In an attempt to determine the chromosomal location of the meningococcal GGI, primers were designed at the ends of 
the GGI sequence, such that they would extend outward. We hypothesized that if a specific primer extension product could be generated with incorporated fluorescent nucleotides (as is done using random primers for CGH), then the location of the GGI could be determined based on the gene probes that hybridize to the specific labelled product. Unfortunately, the use of specific primers in the CGH protocol produced non-specific hybridizations. This is probably due to the temperature necessary for Klenow extension $\left(37^{\circ} \mathrm{C}\right)$, leading to non-specific annealing of the primers. It was noted, however, that the genes of the GGI were the brightest of the features on the microarray following this labelling procedure - which was the opposite of the predicted result. This led us to speculate whether the GGI was present as an extrachromosomal circle, which could account for outward pointing primers producing signal for internal genes.

\section{Knock-out mutants of the GGI}

N. gonorrhoeae strains FA1090 and FA19 and N. meningitidis strain 98/250521 were transformed with PCR products generated using specific primers (GGIdelF and GGIdelR, Table 1) and pGGIdelP recakan or pGGIdelaphA-3 as the templates. Kanamycin-resistant colonies were obtained for all three strains following transformation, and colony PCR using kanamycin cassette directed primers produced amplicons of the expected sizes. Transformants of strains FA19 and 98/250521 were grown overnight without selection for
DNA preparation. Hybridization to the pan-Neisseria microarray-v2 indicated that the GGI genes are still present in these strains, further suggesting that the GGI genes were present in more than one location.

\section{Sequencing demonstrates the chromosomal and extrachromosomal nature of the GGI}

Given the observations suggesting both chromosomal and additional extrachromosomal locations for the GGI, primers were designed to generate products that would be specific and confirm the presence of this element in both states. Sequencing from $N$. gonorrhoeae strain MS11 (GenBank accession nos DQ171943 and DQ171944) joined both ends of the established GGI sequence (AY803022) to the $N$. gonorrhoeae strain FA1090 genome sequence (AE004969) at the dif site (Figs 1 and 2) identified previously (Hamilton et al., 2005). In contrast to our previous observations, these sequence data (GenBank accession no. DQ171943) revealed that the strain FA1090 hypothetical gene XNG0741 is present in strain MS11 and that the GGI has inserted within the MMEung at the dif site (Fig. 1), rather than according to the MME model (Snyder et al., 2004). However, the variation in gene presence within this MME between strains FA1090, MC58, and FAM18 would still classify this location as an MME.

Hybridization of these GGI-containing N. meningitidis strains to the genes within the N. meningitidis strain MC58

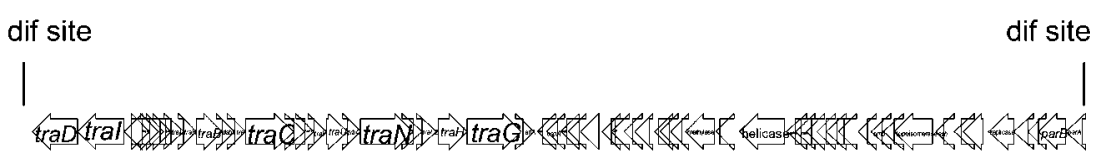

\section{Gonococcal genetic island $57358 \mathrm{bp}$}

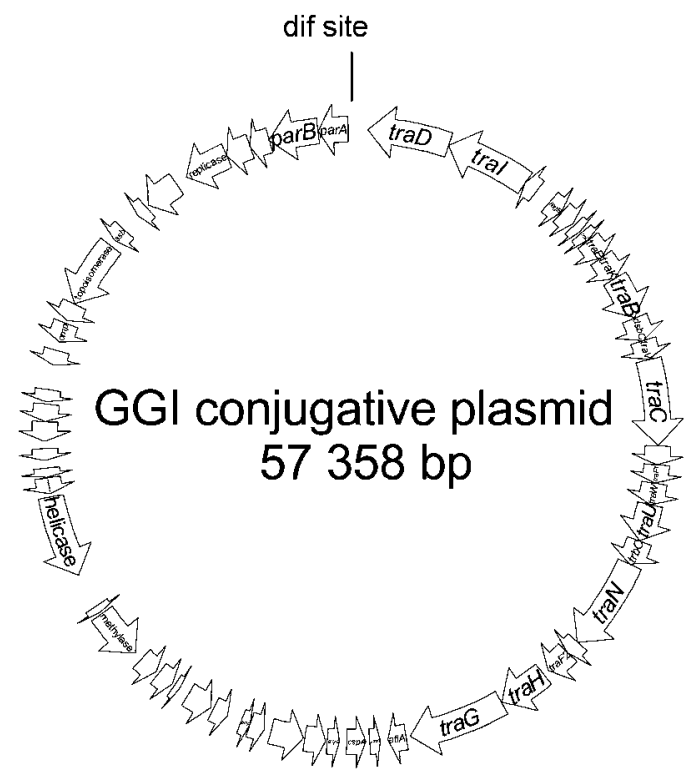

Fig. 2. The GGI is present both in the chromosome and as an extrachromosomal plasmid. Sequencing of $N$. gonorrhoeae strain MS11 using primers within ung (GenBank accession no. DQ171943) and XNG0740 (GenBank accession no. DQ171944) confirmed its location in the chromosome (see Fig. 1), having integrated at the dif site between XNG0741 and XNG0740. Sequencing of a PCR product generated with primers pointing out from the ends of the GGl revealed that this element is also present as an extrachromosomal circle (GenBank accession no. DQ171945). The dif site serves as the junction between the beginning and end of the GGI sequence (GenBank accession no. AY803022). 
MMEung (Snyder et al., 2004) suggests that this region is similar to that of the meningococci, rather than the gonococci (Fig. 1). In N. meningitidis, the dif site is within the annotated hypothetical protein coding region NMB1224. Sequencing of $N$. meningitidis strain A22 using primers WGGIdifR and GGI56out1 revealed that this region is similar to the MMEung in N. meningitidis strain MC58 and that the GGI has inserted at the dif site (Fig. 1) at this location (GenBank accession no. DQ171942).

Sequencing of the strain MS11 amplicon generated using primers GGI01out1 and GGI57out3 (GenBank accession no. DQ171945) confirmed the existence of a circularized form of the GGI in which the two 'outward' ends are joined at the dif site (Fig. 2).

\section{DISCUSSION}

$N$. meningitidis serogroup $\mathrm{H}$ strain $98 / 250521$ and $N$. meningitidis serogroup $\mathrm{W}-135$ strain A22 hybridize to nearly all of the probes to the GGI. These are the first meningococcal strains to be identified that contain the GGI, albeit one that may be incomplete in comparison to the model GGI from $N$. gonorrhoeae strain MS11. atlA, the GGI parA, and a conserved hypothetical gene (GGI42) are apparently absent or divergent from both of these meningococcal strains. The probes for atlA and the conserved hypothetical gene did not hybridize to any of the meningococcal strains, while the GGI parA probe only hybridized to one of the strains $(01 / 241471)$.

Differential presence of genes within the GGI has been previously noted and is the basis for the defined classes of GGI (Dillard \& Seifert, 2001). Class I GGIs contain the genes that have been sequenced from N. gonorrhoeae strain MS11 (GenBank accession no. AY803022) (Dillard \& Seifert, 2001; Dillard \& Hamilton, 2002; Hamilton et al., 2005). Class II GGIs contain the sac-4 allele of traG. The absence of atlA from the GGI of some gonococcal strains has been reported previously as class III GGIs and is associated with an alternative sequence present in the equivalent location, although its nature has not been determined (Dillard \& Seifert, 2001). The absence of atlA in these meningococcal versions of the GGI suggests that they belong to class III. It would be interesting, therefore, to compare a class III gonococcal GGI with those from N. meningitidis strains 98/ 250521 and A22. In addition, all but one of the strains lacked hybridization to the GGI parA probe; therefore, this may indicate either a meningococcal or a GGI class III-specific variation in this sequence, particularly since the associated GGI parB gene is present in these strains and sequencing from strain A22 indicated that at least a portion of parA is present (GenBank accession no. DQ171942).

The previous classification of the GGI as specific to $N$. gonorrhoeae was based on Southern blots conducted using just two hybridization probes: to atlA and traG, both of which were known to be variably present in the GGIs (Dillard \& Seifert, 2001). The absence of the atlA gene in class III GGIs of N. gonorrhoeae is also seen in the meningococcal strains; therefore, the use of this gene as a probe would have prevented identification of any class III GGIs in the strains tested. Further, class II GGIs contain a divergent allele of $\operatorname{tra} G(s a c-4)$, therefore a $\operatorname{tra} G$ probe would not necessarily have identified strains with class II GGIs. The $N$. meningitidis strains tested previously were of serogroups A, B, C, and D (Dillard \& Seifert, 2001). Our microarray hybridizations with DNA from these serogroups also did not reveal GGI sequences.

Although probes on the pan-Neisseria microarray are designed to the most conserved regions of the genes where multiple sequences of individual genes are available, there was only one GGI sequence available for design, from $N$. gonorrhoeae strain MS11. If there are differences (probably greater than at least $20 \%$ divergence) between different allelic versions of genes, then this would lead to the lack of hybridization to the probe, and detection in this system. Specific primer pairs designed using the $N$. gonorrhoeae strain MS11 island sequence frequently failed to generate specific PCR products from meningococcal templates, further indicating divergence of these sequences.

If the GGI is contiguous and present in the same location in the N. meningitidis strains as it is in N. gonorrhoeae strain MS11, then generation of knock-out mutants of the entire GGI would make this clear when the parent and mutant DNA are hybridized to the microarray. However, after preparation of DNA from the transformants and hybridization to the microarray, it was revealed that the genes of the GGI are still present. The same was true for the N. gonorrhoeae strain FA19 transformants; therefore, sequencing was conducted to confirm the chromosomal location of the GGI. It was noted previously that an artificial construct containing the dif site readily excised from the chromosome (Hamilton et al., 2005). If the reverse is true, it may be that the GGI reintegrated at the dif site that is still present in the mutant constructs.

Sequence data revealed that, in strain MS11, the GGI is present both within the chromosome and as an extrachromosomal circular DNA element (Fig. 2). The genes of the GGI share significant similarity with the genes of the F plasmid (Hamilton et al., 2005), therefore its presence as a free and as a chromosomally integrated plasmid is, on this basis, to be expected. The type IV secretion system encoded by the GGI has been shown to secrete chromosomal DNA, much the same as other integrated conjugative plasmids. Unlike most conjugation systems, cell-cell contact is not required, although this may be because it is not necessary in the naturally competent Neisseria species. It has additionally been shown that this secreted DNA is capable of neisserial transformation (Hamilton et al., 2005). Indeed, a neisserial conjugative plasmid was identified previously, but while the GGI is $57 \mathrm{~kb}$, the conjugative plasmid was reported as being $37 \mathrm{~kb}$ (Elwell \& Falkow, 1977). These may represent different unrelated plasmids, or it is possible that these are variants of the GGI plasmid. 


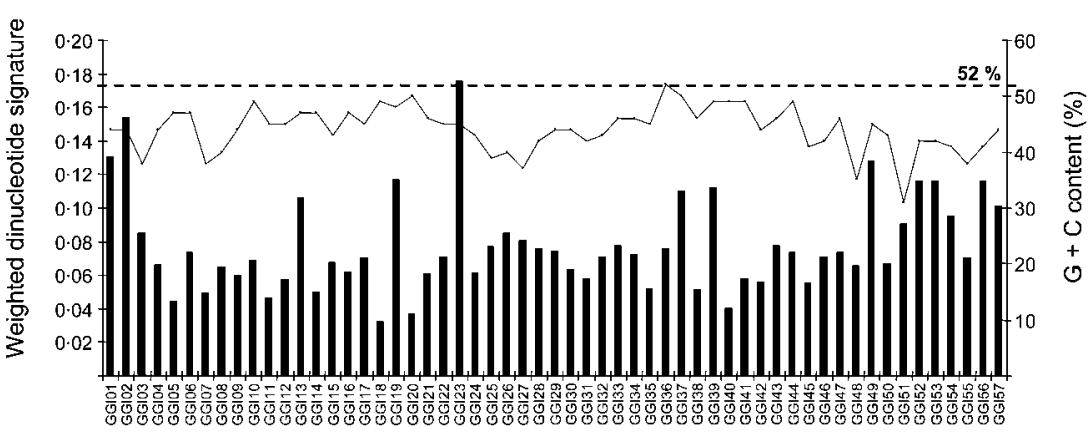

N. gonorrhoeae strain MS11 GGI
Fig. 3. Dinucleotide signature analysis and $\mathrm{G}+\mathrm{C}$ content of the $N$. gonorrhoeae strain MS11 GGI. Each annotated feature is plotted along the $x$ axis, with the dinucleotide signature weighted deviation from the genome signature (bars) on the left $y$ axis and the $\mathrm{G}+\mathrm{C}$ content of each gene (solid line) on the right $y$ axis. The dotted line delineates $52 \%$, the mean neisserial $\mathrm{G}+\mathrm{C}$ content.
In its circularized form, the adjacent coding regions of the GGI are now orientated in the same direction. Strand switching for the coding regions occurs only between GGI03 and iagB (GGI04) and between atlA (GGI24) and cspA (GGI26) (Fig. 2). While as an integrated island, the organization is less structured, in the circularized form, the global organization of the genes and potential transcriptional units is more coherent.

The absence of some of the GGI genes in different strains suggests either that the complete GGI has developed from the combination of distinct segments, or that portions of it have been deleted or added. Compared with the genome sequence, there are distinct differences in the $\mathrm{G}+\mathrm{C}$ content and dinucleotide signatures of the genes of the GGI of $N$. gonorrhoeae strain MS11, which suggests that these genes may have had a foreign origin (Fig. 3). However, it also shows features of considerable amelioration, or having origins not greatly divergent in composition, that suggest that it has been present within the Neisseria species for a relatively long time. A 'foreign' origin is also consistent with the absence of the common neisserial intergenic Correia repeat (Correia et al., 1986, 1988), but it may also be that these elements are not maintained on neisserial plasmids.

\section{ACKNOWLEDGEMENTS}

This project and LASS are supported by a Wellcome Trust Project Grant awarded to N. J. S. Simon McGowan of the Sir William Dunn School of Pathology/Weatherall Institute of Molecular Medicine Computational Biology Research Group provided bioinformatics support for this project. We would like to thank Joe Dillard for the provision of the $N$. gonorrhoeae strain MS11 GGI sequence used in the construction of the probes for the pan-Neisseria microarray. The panNeisseria microarray-v2 made use of the genome sequence of $N$. meningitidis strain FAM18. These sequence data were produced by the N. meningitidis serogroup C strain FAM18 Sequencing Group at the Sanger Institute and can be obtained from ftp://ftp.sanger.ac.uk/pub/ pathogens $/ \mathrm{nm}$. The original pan-Neisseria microarray made use of the genome sequence of N. gonorrhoeae strain FA1090 (GenBank accession no. AE004969).

\section{REFERENCES}

Bonfield, J. K., Beal, K. F., Betts, M. J. \& Staden, R. (2002). Trev: a DNA trace editor and viewer. Bioinformatics 18, 194-195.

Correia, F. F., Inouye, S. \& Inouye, M. (1986). A 26-base-pair repetitive sequence specific for Neisseria gonorrhoeae and Neisseria meningitidis genomic DNA. J Bacteriol 167, 1009-1015.

Correia, F. F., Inouye, S. \& Inouye, M. (1988). A family of small repeated elements with some transposon-like properties in the genome of Neisseria gonorrhoeae. J Biol Chem 263, 12194-12198.

Dillard, J. P. \& Hamilton, H. L. (2002). Sequence and mutational analysis of the gonococcal genetic island. In Abstracts of the Thirteenth International Pathogenic Neisseria Conference, p. 79. Edited by D. A. Caugant \& E. Wedege. Oslo: Nordberg Aksidenstrykkeri AS.

Dillard, J. P. \& Seifert, H. S. (1997). A peptidoglycan hydrolase similar to bacteriophage endolysins acts as an autolysin in Neisseria gonorrhoeae. Mol Microbiol 25, 893-901.

Dillard, J. P. \& Seifert, H. S. (2001). A variable genetic island specific for Neisseria gonorrhoeae is involved in providing DNA for natural transformation and is found more often in disseminated infection isolates. Mol Microbiol 41, 263-277.

Elwell, L. P. \& Falkow, S. (1977). Plasmids of the genus Neisseria. In The Gonococcus, pp. 138-154. Edited by R. B. Roberts. New York: Wiley.

Hamilton, H. L., Domínguez, N. M., Schwartz, K. J., Hackett, K. T. \& Dillard, J. P. (2005). Neisseria gonorrhoeae secretes chromosomal DNA via a novel type IV secretion system. Mol Microbiol 55, 1704-1721.

Kellogg, D. S., Jr, Peacock, W. L., Jr, Deacon, W. E., Brown, L. \& Pirkle, C. I. (1963). Neisseria gonorrhoeae. I. Virulence genetically linked to clonal variation. J Bacteriol 85, 1274-1279.

McAllister, C. F. \& Stephens, D. S. (1993). Analysis in Neisseria meningitidis and other Neisseria species of genes homologous to the FKBP immunophilin family. Mol Microbiol 10, 13-23.

McShan, W. M., Williams, R. P. \& Hull, R. A. (1987). A recombinant molecule from a disseminating strain of Neisseria gonorrhoeae that confers serum bactericidal resistance. Infect Immun 55, 3017-3022.

Ménard, R., Sansonetti, P. \& Parsot, C. (1994). The secretion of the Shigella flexneri Ipa invasins is activated by epithelial cells and controlled by IpaB and IpaD. EMBO J 13, 5293-5302.

Parkhill, J., Achtman, M., James, K. D. \& 25 other authors (2000). Complete DNA sequence of a serogroup A strain of Neisseria meningitidis Z2491. Nature 404, 502-506.

Saunders, N. J. \& Snyder, L. A. S. (2002). The minimal mobile element. Microbiology 148, 3756-3760. 
Saunders, N. J., Boonmee, P., Peden, J. F. \& Jarvis, S. A. (2005). Inter-species horizontal transfer resulting in core-genome and nicheadaptive variation within Helicobacter pylori. BMC Genomics 6, 9.

Snyder, L. A. S., Saunders, N. J. \& Shafer, W. M. (2001). A putatively phase variable gene $(d c a)$ required for natural competence in Neisseria gonorrhoeae but not Neisseria meningitidis is located within the division cell wall $(\mathrm{dcw})$ gene cluster. J Bacteriol 183, 1233-1241.
Snyder, L. A. S., Davies, J. K. \& Saunders, N. J. (2004). Microarray genomotyping of key experimental strains of Neisseria gonorrhoeae reveals gene complement diversity and five new neisserial genes associated with Minimal Mobile Elements. BMC Genomics 5, 23.

Tettelin, H., Saunders, N. J., Heidelberg, J. \& 39 other authors

(2000). Complete genome sequence of Neisseria meningitidis serogroup B strain MC58. Science 287, 1809-1815. 